\title{
Limites de l'éthique et action politique
}

\section{Gérald Berthoud}

\section{(2) OpenEdition}

Journals

Édition électronique

URL : http://journals.openedition.org/ress/681

DOI : $10.4000 /$ ress.681

ISSN : 1663-4446

\section{Éditeur}

Librairie Droz

\section{Édition imprimée}

Date de publication : 1 août 2000

Pagination : 5-6

ISBN : 2-600-00450-5

ISSN : 0048-8046

\section{Référence électronique}

Gérald Berthoud, «Limites de l'éthique et action politique », Revue européenne des sciences sociales [En ligne], XXXVIII-118 | 2000, mis en ligne le 15 décembre 2009, consulté le 22 septembre 2020. URL : http://journals.openedition.org/ress/681 ; DOI : https://doi.org/10.4000/ress.681 


\section{Gérald BERTHOUD}

\section{LIMITES DE L'ÉTHIQUE ET ACTION POLITIQUE*}

\section{Objectifs}

Aujourd'hui, à des degrés variables, toutes les sociétés sont entraînées dans la voie d'un capitalisme vu par beaucoup comme une «révolution perpétuelle». Pour penser ce mouvement dans toute sa force et dans toute son extension, il est nécessaire de l'envisager comme une transformation radicale de l'homme, de la société et du monde, comprenant, de manière irréductible, les domaines de la science, de la technique et de l'économie. L'objectif ultime n'est-il pas de tout savoir, de tout faire et de tout avoir? Potentiellement sans fin et pourtant toujours limité de fait, ce mouvement est à la fois un inachèvement continuel et une constante négation de toute frontière. Par sa puissance même, il tente d'orienter, à des degrés divers, toutes les idées et toutes les actions vers un monde toujours plus instrumentalisé comme la condition même d'une libération individuelle et d'un bien-être insatiable.

Autant dire qu'il semble difficile de tracer des limites, voire impossible d'en établir, dans un contexte vu comme mouvant, fluide et instable. Pourtant ne sommes-nous pas confrontés toujours davantage à des situations dans lesquelles des limites semblent être atteintes, voire dépassées? De manière inexorable, des interrogations sur l'admissible ou l'inadmissible, le souhaitable ou ce qu'il faudrait éviter à tout prix ne peuvent plus être éludées. Qu'il s'agisse des limites écologiques, démographiques, économiques, ou des limites qui sont induites par diverses formes d'inégalité; ou encore des limites intérieures ou biologiques, celles des interventions dans le corps humain, le recours à une réflexion éthique, propre à fonder la légitimité des actions, apparaît comme une nécessité inéluctable.

Mais les contradictions et les paradoxes affleurent immédiatement. Science, technique et économie ne se reconnaissent pas de limites, car ce serait, pour ces trois domaines entraînés dans un même mouvement d'efficacité et de rentabilité, une manière de se nier. Dans un monde où l'innovation technique et économique est érigée en une valeur centrale, ce qui est illégitime aujourd'hui ne le sera sans doute plus demain. Le clonage humain, il y a encore peu jugé comme inacceptable, est sur le point de se légitimer, en invoquant son utilité thérapeutique et médicale.

* Texte envoyé aux participants du XVI e colloque annuel du Groupe d'Étude «Pratiques sociales et Théories », en vue de préparer leur communication. Intitulée «Limites de l'éthique et action politique», cette rencontre s'est déroulée les 2 et 3 décembre 1999 à l’Université de Lausanne. 
Comment concevoir une éthique qui ne soit pas un simple alibi dans une culture utilitaire, qui justifie à l'avance les découvertes de la science, les inventions de la technique et les produits de l'économie? Et pourtant l'éthique, si elle ne s'identifie pas à un recueil de règles abstraites et de commandements universels, devrait apporter une contribution essentielle, en vue de penser les problèmes du monde actuel. Mais l'éthique des limites se heurte très vite aux limites de l'éthique. Cette dernière postule que les pratiques sociales sont légitimes dans la mesure où elles sont fondées sur des principes justifiables et plausibles. Or une telle réflexion éthique néglige le fait qu'un accord sur les principes devrait s'appuyer avant tout sur des pratiques et des conventions sociales partagées. Le danger est grand d'ignorer que dans la vie sociale les principes doivent être appliqués dans des situations particulières. Déterminer les croyances éthiques correctes, en vue de fixer des systèmes d'obligations catégoriques, revient à oublier que les devoirs découlent de notre participation à des rapports sociaux spécifiques. Ce que la conception moderne de la vie sociale tend à occulter, en imposant la moralité universelle de l'obligation catégorique.

Plus encore, les problèmes du monde actuel dépassent la seule éthique personnelle. Promouvoir alors une éthique collective n'est envisageable que pour autant que nous posions la question des rapports entre éthique et politique. Aussitôt une difficulté majeure surgit. La pensée moderne n'a-t-elle pas établi une séparation entre l'éthique et la politique, au point de concevoir cette dernière comme un domaine de l'amoralité.

Certes, la politique doit satisfaire l'exigence primordiale de garantir l'ordre, en vue de rendre possible la vie en commun. Soumise à un processus d'autonomisation, comme les autres domaines de la vie collective, la politique doit remplir sa fonction spécifique de manière efficace. Mais elle doit aussi répondre aux demandes de liberté, d'égalité, de fraternité et de justice. Il y a là une double conception de la politique, vue à la fois comme une technique, pour élaborer et imposer des règles et des normes, et comme une instance en mesure de promouvoir des fins.

La politique, conçue de cette manière, est en crise aujourd'hui. Une situation qui favorise les spéculations éthiques et qui tend à imposer l'idée que l'éthique serait au-dessus de la politique. Faut-il admettre alors qu'un débat critique sur l'éthique devrait nécessairement déboucher sur une conception renouvelée de la politique? Et admettre aussi, contre le conformisme ambiant, que la raison d'être de la politique ne se réduit pas à assurer les conditions d'un bien-être généralisé?

Institut d' anthropologie et de sociologie

Université de Lausanne 